\title{
Sigma models for genuinely non-geometric backgrounds
}

\author{
Larisa Jonke* \\ Division of Theoretical Physics, Rudjer Bošković Institute, \\ Bijenicka 54, 10000 Zagreb, Croatia \\ E-mail: larisa@irb.hr
}

In this short note we advocate for the usefulness of generalized geometry and in particular of the Courant algebroid structure in addressing the issue of "non-geometry" in string theory. This mathematical framework allows for a unified description of gauge symmetries and diffeomorphisms and naturally incorporates background fluxes of string theory. Using these tools we propose an extension of known membrane sigma models which is suitable for the description of truly nongeometric backgrounds.

Proceedings of the Corfu Summer Institute 2015 "School and Workshops on Elementary Particle Physics and Gravity"

1-27 September 2015

Corfu, Greece

${ }^{*}$ Speaker. 


\section{Introduction}

Understanding of the quantum structure of space-time requires new concepts and tools for the description of geometry and symmetries of physical theories relevant at energies and distances where both gravity and quantum physics play roles. Attempts to combine general relativity and quantum mechanics at the Plank scale indicate that our classical description of space-time as a smooth manifold should be replaced by some kind of fuzzy or foam-like structure in that regime. As a consequence, we are forced to abandon the idea of point particles as fundamental degrees of freedom and introduce non-local excitations like strings and higher-dimensional branes. This immediately raises the question of symmetries encompassing the dynamics of these non-local degrees of freedom. In particular, one needs to rethink the idea of gauge symmetry and its interplay with space-time symmetries in theories where space-time is dynamical.

This serves as a motivation to look for a framework that would allow for a unified treatment of diffeomorphisms and gauge symmetries, and one such possibility is generalized complex geometry as introduced by Hitchin [1] and worked out by Gualtieri [2]. The starting point in the generalized geometry (GG) framework is an extension of the tangent bundle to the socalled generalized tangent bundle $\mathbb{T M}=\mathrm{TM} \oplus \mathrm{T}^{\star} \mathrm{M}$, the sections of which are now $\mathfrak{X} \in \Gamma(\mathbb{T M})$ : $\mathfrak{X}=X+\eta, X \in \Gamma(\mathrm{TM}), \eta \in \Gamma\left(\mathrm{T}^{\star} \mathrm{M}\right)$, i.e., the sum of a vector $X$ and 1 -form $\eta$. Our interest in the GG framework lies in the fact that the symmetries of the extended bundle include both diffeomorphisms and gauge transformations which are now part of $\mathrm{O}(\mathrm{d}, \mathrm{d})$ transformations. One is reminded that the latter is related to the T-duality group of string theory compactified on a d-dimensional torus.

In general, duality symmetries are means to identify apparently different physical settings. We are familiar with examples of duality in field theories, where one uses duality to relate differently parametrized spaces of fields/solutions of the underlying theory. In this respect, it shouldn't be surprising that dualities in string theory (where geometric degrees of freedom are dynamical objects in the theory) can identify apparently distinct geometries. What is interesting is that T-duality transformations uncovered the existence of string-theory backgrounds that cannot be described by standard geometry. In particular, metric and background fluxes such as NSNS flux, torsion or geometric flux and RR fluxes after T-duality transformations (which is a symmetry of the theory) could not be understood in terms of usual geometry [3-6]. It turned out that this new geometry (sometimes called non-geometry) could be described with techniques from the differential geometry of Lie and Courant algebroids [7-12] which are also used in the GG framework mentioned above. In short, while the symmetries of the generalized tangent bundle naturally incorporate diffeomorphisms and gauge transformation, the structure of algebroid (Courant in particular) naturally incorporates background fields of string theory.

In this short note based on Ref. [13] we would like to advocate for the usefulness of the described framework in addressing the issue of "non-geometry" in string theory. For this purpose we will introduce structures necessary for defining Courant algebroids. In the next section we follow Roytenberg's construction based on protobialgebroids [14], as it naturally includes all stringtheory fluxes. We then relate these structures with bosonic string sigma models using the result [15] that for every Courant algebroid one can construct a topological sigma model of the type introduced by Alexandrov, Kontsevich, Schwarz and Zaboronsky (AKSZ) [16]. Finally, we argue that one has 
to go beyond this construction to capture "non-geometry" originating in T-duality. Motivated by a similar approach adopted in Ref. [10], we propose an extension of the sigma models with standard target space to ones with phase space as target. The relation between our 3D/2D perspective and the doubled formalism of string theory [17] and double field theory (DFT) [18-21] is still not clear although there are intriguing similarities.

\section{Courant algebroid in a nutshell}

The Courant algebroid (CA) is a vector bundle over a manifold, with a bracket defined on its sections, a symmetric bilinear form, an anchor map from the bundle to the tangent bundle of the manifold with certain compatibility conditions between these structures. The precise definition is given in the Appendix. One perhaps more illuminating construction of CAs we are following here goes through protobialgebroids as defined in Ref. [14]. We start with a vector bundle $L$ and its dual $L^{\star}$ with closed bracket and anchor map on each of them and a pair of generalized 3-forms $\phi \in \Gamma\left(\wedge^{3} L^{\star}\right)$ and $\psi \in \Gamma\left(\wedge^{3} L\right)$ such that

- $[X, f Y]_{L}=f[X, Y]_{L}+(\rho(X) f) Y$, and $[\eta, f \xi]_{L^{\star}}=f[\eta, \xi]_{L^{\star}}+\left(\rho_{\star}(\eta) f\right) \xi, f \in C^{\infty}(M)$,

- $\rho\left([X, Y]_{L}\right)=[\rho(X), \rho(Y)]_{\mathrm{Lie}}+\rho_{\star} \phi(X, Y, \cdot)$, and $\rho_{\star}\left([\eta, \xi]_{L^{\star}}\right)=\left[\rho_{\star}(\eta), \rho_{\star}(\xi)\right]_{\text {Lie }}+\rho \psi(\eta, \xi, \cdot)$,

- $\operatorname{Jac}[X, Y, Z]_{L}=\mathrm{d}_{L^{\star}} \phi(X, Y, Z)+\phi\left(\mathrm{d}_{L^{\star}} X, Y, Z\right)+\phi\left(X, \mathrm{~d}_{L^{\star}} Y, Z\right)+\phi\left(X, Y, \mathrm{~d}_{L^{\star}} Z\right)$, and $\left[[\eta, \xi]_{L^{\star}}, \omega\right]_{L^{\star}}+$ c.p. $=\mathrm{d}_{L} \psi(\eta, \xi, \omega)+\psi\left(\mathrm{d}_{L} \eta, \xi, \omega\right)+\psi\left(\eta, \mathrm{d}_{L} \xi, \omega\right)+\psi\left(\eta, \xi, \mathrm{d}_{L} \omega\right)$.

- $\mathrm{d}_{L} \phi=0$ and $\mathrm{d}_{L^{\star}} \psi=0$,

where $X, Y, Z \in \Gamma(L)$ and $\eta, \xi, \omega \in \Gamma\left(L^{\star}\right)$. These four properties are generalizations of familiar properties of the tangent bundle and are realized with the aid of anchor maps. The first property is just a Leibniz rule for each bundle, while the second one says that the anchor maps are homomorphisms twisted by generalized 3-forms. The third property is a twisted version of the Jacobi identity - in general the brackets on the corresponding bundles are not Lie brackets, in particular in presence of generalized 3-forms. The last property states that the generalized 3-forms which we want to relate to string-theory fluxes are closed. The derivations on each bundle are again simple generalization of the standard exterior derivative obtained with the aid of the anchor map. In particular, they are simply defined as maps $\mathrm{d}_{L}: \Gamma\left(\wedge^{p} L^{\star}\right) \rightarrow \Gamma\left(\wedge^{p+1} L^{\star}\right)$ and $\mathrm{d}_{L^{\star}}: \Gamma\left(\wedge^{p} L\right) \rightarrow \Gamma\left(\wedge^{p+1} L\right)$, acting as follows [22]:

$$
\begin{aligned}
\mathrm{d}_{L} \omega\left(X_{1}, \ldots, X_{p+1}\right)= & \sum_{i=1}^{p+1}(-1)^{i+1} \rho\left(X_{i}\right) \omega\left(X_{1}, \ldots, \hat{X}_{i}, \ldots, X_{p+1}\right)+ \\
& +\sum_{i<j}(-1)^{i+j} \omega\left(\left[X_{i}, X_{j}\right]_{L}, X_{1}, \ldots, \hat{X}_{i}, \ldots, \hat{X}_{j}, \ldots, X_{p+1}\right), \\
\mathrm{d}_{L^{\star}} \Omega\left(\eta_{1}, \ldots, \eta_{p+1}\right)= & \sum_{i=1}^{p+1}(-1)^{i+1} \rho_{\star}\left(\eta_{i}\right) \Omega\left(\eta_{1}, \ldots, \hat{\eta}_{i}, \ldots, \eta_{p+1}\right)+ \\
& +\sum_{i<j}(-1)^{i+j} \Omega\left(\left[\eta_{i}, \eta_{j}\right]_{L^{\star}}, \eta_{1}, \ldots, \hat{\eta}_{i}, \ldots, \hat{\eta}_{j}, \ldots, \eta_{p+1}\right),
\end{aligned}
$$


for arbitrary generalized $p$-forms $\omega \in \Gamma\left(\wedge^{p} L^{\star}\right)$ and $\Omega \in \Gamma\left(\wedge^{p} L\right)$.

Given these data one can construct a Courant algebroid on $E=L \oplus L^{\star}$; in particular, the bracket of the CA is

$$
\begin{aligned}
{[X+\eta, Y+\xi]_{E}=} & {[X, Y]_{L}+\mathscr{L}_{X} \xi-\mathscr{L}_{Y} \eta-\frac{1}{2} \mathrm{~d}_{L}(X(\xi)-Y(\eta))+} \\
& +[\eta, \xi]_{L^{\star}}+\mathscr{L}_{\eta} Y-\mathscr{L}_{\xi} X+\frac{1}{2} \mathrm{~d}_{L^{\star}}(X(\xi)-Y(\eta))- \\
& -\phi(X, Y, \cdot)-\psi(\eta, \xi, \cdot)
\end{aligned}
$$

and the anchor of the CA is the sum of anchors of the dual bundles:

$$
a(X+\eta)=\rho(X)+\rho_{\star}(\eta) .
$$

In this construction the natural bilinear symmetric form on the CA is given by the duality relation between the vector bundles $L$ and $L^{\star}$ :

$$
\langle X+\eta, Y+\xi\rangle_{E}=\frac{1}{2}(X(\xi)+Y(\eta))
$$

Using these identifications and the protobialgebroid data one can easily confirm that the compatibility conditions for these structures as given in the Appendix are satisfied.

We choose this particular construction of CAs motivated by its apparent "doubled" structure, having in mind physical instances of doubling like phase space, duality symmetries, double field theory etc. Note however that although Roytenberg showed [14] that for every protobialgebroid there exists an associated CA, it is not necessary that there exist a (proto)bialgebroid structure underlying a generic Courant algebroid as defined in the Appendix.

\section{Membrane sigma model}

As discussed by Roytenberg [15] we know that every Courant algebroid has an associated (topological) sigma model of the type described by Alexandrov, Kontsevich, Schwarz and Zaboronsky (AKSZ) in Ref. [16]. The AKSZ master action contains fields with ghost number 0, 1, 2 and 3 , but here we focus on the 0 -ghost sector:

$$
S_{\Sigma_{3}}[X, A, F]=\int_{\Sigma_{3}}\left(F_{a} \wedge \mathrm{d} X^{a}+\frac{1}{2} \eta_{I J} A^{I} \wedge \mathrm{d} A^{J}-P_{I}^{a} A^{I} \wedge F_{a}+\frac{1}{6} T_{I J K} A^{I} \wedge A^{J} \wedge A^{K}\right) .
$$

This is a membrane topological action in 3D, and its relation with the CA can be seen as follows. The indices $I, J$ are Courant algebroid indices, while the index $a$ is a curved index. $X^{a}$ are the world volume scalars on the membrane, or the components of the map $X: \Sigma_{3} \rightarrow \mathrm{M}$, M being the target spacetime. $A^{I}$ is valued in $\Omega^{1}\left(\Sigma_{3}, X^{\star} E\right)$, where $X^{\star}$ denotes the pull back with respect to the world volume scalar fields. $F_{a}$ is an auxiliary world volume 2 -form in $\Omega^{2}\left(\Sigma_{3}, X^{\star} \mathrm{T}^{\star} \mathrm{M}\right)$ that will be integrated out in the reduced string model. Moreover, $\eta$ is the $\mathrm{O}(\mathrm{d}, \mathrm{d})$ invariant metric given as symmetric bilinear form of the CA, namely

$$
\eta_{I J}=\left(\begin{array}{cc}
0 & \mathbf{1}_{d} \\
\mathbf{1}_{d} & 0
\end{array}\right),
$$


and $P_{I}^{a}$ is the anchor matrix defined through the relation

$$
a\left(\mathfrak{X}_{I}\right)=P_{I}^{a}(X) \partial_{a},
$$

where $a: E \rightarrow \mathrm{TM}$ is the anchor of the CA. Finally, $T \in \Omega^{3}\left(\Sigma_{3}, X^{\star} E\right)$ is the generalized 3-form we introduced in the previous section.

Having in mind physical applications we assume that the manifold $\Sigma_{3}$ has a boundary, say $\partial \Sigma_{3}:=\Sigma_{2}$, and there we add a general topological boundary term as in Ref. [8] (see also [10,23]):

$$
S_{\partial \Sigma_{3}, \text { top }}=\int_{\Sigma_{2}} \frac{1}{2} \mathscr{B}_{I J}(X) A^{I} \wedge A^{J}
$$

More explicitly, with the splitting $A^{I}=\left(q^{i}, p_{i}\right)$,

$$
\frac{1}{2} \mathscr{B}_{I J}(X) A^{I} \wedge A^{J}=\frac{1}{2} \mathscr{B}_{i j}(X) q^{i} \wedge q^{j}+\frac{1}{2} \mathscr{B}^{i j}(X) p_{i} \wedge p_{j}+\frac{1}{2} \mathscr{B}_{j}^{i}(X) q^{j} \wedge p_{i}
$$

The membrane sigma model with boundary terms comes with a set of consistency conditions. In particular, the boundary conditions should match the equations of motion on the boundary. The second condition imposes the vanishing of the sector of the bulk 3D action that does not reduce to the boundary via the field equations. This condition follows from the classical master equation [23]. These bulk/boundary consistency conditions allow for a systematic characterization of fluxes and can be identified as integrability conditions for Dirac structures. A Dirac structure is a maximal isotropic and involutive sub-bundle of a CA; its rank is half of that of the CA and it is closed under the CA bracket. One can find the explicit relations in Ref. [13].

Application of the above action in string theory suggests that we integrate out the auxiliary field from the $3 \mathrm{D}$ action and focus on the 2D reduced theory. There one should also add dynamics thus breaking the topological nature of the theory, see [13] for more details. However, the important lesson we learn from the analysis of the 2D reduced theory, already inferred in $[8,10]$, is that this approach does neither describe all non-geometric solutions obtained through T-duality transformation on geometric ones nor genuine non-geometric backgrounds.

\section{One step beyond}

In order to motivate the generalization of the framework described above here we recall the example worked out in Ref. [10] ${ }^{1}$. Consider the following membrane sigma model on a torus and choose $T=R$ as only generalized 3-form with $R$ a constant 3-vector. The topological 3D action is

$$
S_{R}[X, A, F]=\int_{\Sigma_{3}}\left(F_{a} \wedge \mathrm{d} X^{a}+q^{a} \wedge \mathrm{d} p_{a}-q^{a} \wedge F_{a}+\frac{1}{6} R^{a b c} p_{a} \wedge p_{b} \wedge p_{c}\right),
$$

where we used only early Latin indices as we refer to the flat torus. Integrating out the auxiliary 2-form $F_{a}$ one obtains

$$
S_{R}[X, A, F]=\int_{\Sigma_{2}} p_{a} \wedge \mathrm{d} X^{a}+\int_{\Sigma_{3}} \frac{1}{6} R^{a b c} p_{a} \wedge p_{b} \wedge p_{c} .
$$

\footnotetext{
${ }^{1}$ Attention should however be paid to the fact that there is no underlying CA behind the membrane action for this example.
} 
The equation of motion for $X^{a}$ is simply

$$
\mathrm{d} p_{a}=0
$$

This means that the 1-form $p_{a}$ may be written locally as

$$
p_{a}=\mathrm{d} \tilde{X}_{a},
$$

where $\tilde{X}_{a} \in C^{\infty}\left(\Sigma_{3}, X^{\star} T^{\star} \mathrm{M}\right)$ play role similar to dual coordinates in DFT. As suggested in Ref. [10], these coordinates can be seen as defining an augmented embedding of the 2-dimensional boundary theory on $\Sigma_{2}$ in the full cotangent bundle of the target manifold M. So, from the equation of motion of the sigma model we obtained generalized (or doubled) target space coordinates $\left(\mathbb{X}^{I}\right)=\left(X^{a}, \tilde{X}_{a}\right)$ which correspond to a map $\mathbb{X}=\left(\mathbb{X}^{I}\right): \Sigma_{3} \rightarrow \mathrm{T}^{\star} \mathrm{M}$.

The above doubling could be also understood in the spirit of the topological approach to Tduality [24, 25], which was explained via Courant algebroids in Refs. [26-28]. In this approach there is a product manifold $\mathrm{M} \times \tilde{\mathrm{M}}$ of original and dual spaces and T-duality corresponds to an isomorphism of twisted K-theories [24, 25]. In [27] it was shown that this can be extended to an isomorphism between the corresponding CAs. Here we want to associate $\mathbb{X}^{I}$ to the product manifold $\mathrm{M} \times \tilde{\mathrm{M}}$. Although the precise correspondence needs to be worked out, we propose that the membrane sigma models for CAs over this extended target space should correspond to the ones we will consider below.

The generalized embedding proposed in Ref. [10] is naturally extended in such a way as to allow all the fields that appear in the model to depend both on $X^{a}$ and $\tilde{X}_{a}$. In that case the formulation (3.1) for the sigma model looks rather restrictive. From the viewpoint of physics, Eq. (3.1) does not contain $d \tilde{X}_{a}$ at all, which should not be the case in general. Thus, returning to the general case, we propose the following. First, we allow $B, \beta, h, a$ and $T$ to depend on both $X^{a}$ and $\tilde{X}_{a}$. Second, we introduce another auxiliary world volume 2 -form $\tilde{F}^{a} \in \Omega^{2}\left(\Sigma_{3}, \mathbb{X}^{\star} \mathrm{TM}\right)$ with a vector index. Then we write the 3-dimensional action

$$
S_{\Sigma_{3}}=\int_{\Sigma_{3}}\left(F_{a} \wedge \mathrm{d} X^{a}+\tilde{F}^{a} \wedge \mathrm{d} \tilde{X}_{a}+\frac{1}{2} \eta_{I J} A^{I} \wedge \mathrm{d} A^{J}-P_{I}^{a} A^{I} \wedge F_{a}-\tilde{P}_{a I} A^{I} \wedge \tilde{F}^{a}+\frac{1}{6} T_{I J K} A^{I} \wedge A^{J} \wedge A^{K}\right) .
$$

In more compact notation, writing $P_{I}^{J}=\left(P_{I}^{a}, \tilde{P}_{a I}\right)$ and $F^{I}=\left(F_{a}, \tilde{F}^{a}\right)$ for $F^{I} \in \Omega^{2}\left(\Sigma_{3}, \mathbb{X}^{\star} E\right)$, we get

$$
S_{\Sigma_{3}}=\int_{\Sigma_{3}}\left(\delta_{I J} F^{I} \wedge \mathrm{d} \mathbb{X}^{J}+\frac{1}{2} \eta_{I J} A^{I} \wedge \mathrm{d} A^{J}-\delta_{J K} P_{I}^{J} A^{I} \wedge F^{K}+\frac{1}{6} T_{I J K} A^{I} \wedge A^{J} \wedge A^{K}\right) .
$$

The boundary action has the same form as before, namely

$$
S_{\Sigma_{2}}=\int_{\Sigma_{2}} \frac{1}{2} \mathscr{B}_{I J} A^{I} \wedge A^{J}
$$

but now $\mathscr{B}=\mathscr{B}(X, \tilde{X})$. Furthermore, we introduced the object $\tilde{P}_{a I}$, which was absent before. These are the components of a map $\tilde{P}: E \rightarrow \mathrm{T}^{\star} \mathrm{M}$ that maps elements of the Courant algebroid to the cotangent bundle. Examples of such a map is the unit map on 1-forms and the map $B^{\sharp}: \mathrm{TM} \rightarrow \mathrm{T}^{\star} \mathrm{M}$ that acts simply as $B^{\sharp}\left(X_{i}\right)=B_{i j} \eta^{j}$.

In Ref. [13] we have examined the bulk/boundary consistency conditions and showed how one can solve them in an example. Moreover, one can integrate out both auxiliary two-forms and obtain 
a reduced $2 \mathrm{D}$ action, which now has non-trivial dependence on both sets of coordinates. We suggest that this is the framework within which one could describe genuine non-geometric backgrounds of string theory.

\section{Outlook}

In this note we presented a membrane sigma model that could provide a consistent world-sheet approach to realizing new geometries appearing in string theory. We have shown that at the level of the world-sheet sigma model action one is naturally lead to an apparent doubling of degrees of freedom by careful analysis of equation of motion. Furthermore, the bulk/boundary consistency condition provide a well-defined procedure for obtaining the 2D reduced theory with background fluxes. We have shown that in general this reduced theory depends on both set of coordinates.

Of course, this proposal should be further investigated and two main questions should be answered. The first one is the question of the geometric interpretation of the 3D sigma model and its precise relation to work on topological T-duality. An interesting question in particular is how one can "reduce" the notion of T-duality as isomorphisms of CAs to T-duality on 2D worldsheet. There are results indicating that $2 \mathrm{D}$ gauged sigma models might offer a valuable input in this respect [29-31].

Furthermore, one needs to understand better the physical aspects of the reduced 2D theory; here we need to analyze appropriate string backgrounds and compare with results obtained in other approaches, DFT in particular. Our analysis so far would suggest that there is a relation between the section condition/strong constraint of DFT and the integrability condition for Dirac structures of a CA. However, the precise relation is still unclear to us and should be worked out.

Acknowledgment. This work is based on a collaboration with A. Chatzistavrakidis and O. Lechtenfeld [13]. I would like to thank the organizers of Corfu Summer Institute 2015 for providing me with an opportunity to present these results, and R. Szabo for discussion. This work was supported in part by the Action MP1405 QSPACE from the Europe an Cooperation in Science and Technology (COST). The work of L.J. was supported in part by Croatian Science Foundation under the project IP-2014-09-3258.

\section{Appendix}

For completeness of presentation here we give the definition of a Courant algebroid according to Ref. [32].

A Courant algebroid is a quadruplet $\left(E,[\cdot, \cdot]_{E},\langle\cdot, \cdot\rangle_{E}, a\right)$ of the following data:

- a vector bundle $E$ over M,

- a skew-symmetric bracket on $\Gamma(E)$,

- a non-degenerate symmetric bilinear form on $E$,

- and an anchor map $a: E \rightarrow \mathrm{TM}$, 
such that for $\mathfrak{X}_{i} \in \Gamma(E)$ :

1. $\left[\left[\mathfrak{X}_{1}, \mathfrak{X}_{2}\right]_{E}, \mathfrak{X}_{3}\right]_{E}+$ c.p. $=\mathscr{D} \mathscr{N}\left(\mathfrak{X}_{1}, \mathfrak{X}_{2}, \mathfrak{X}_{3}\right), \quad 3 \mathscr{N}=\left\langle\left[\mathfrak{X}_{1}, \mathfrak{X}_{2}\right]_{E}, \mathfrak{X}_{3}\right\rangle_{E}+$ c.p.,

2. $a\left(\left[\mathfrak{X}_{1}, \mathfrak{X}_{2}\right]_{E}\right)=\left[a\left(\mathfrak{X}_{1}\right), a\left(\mathfrak{X}_{2}\right)\right]_{\text {Lie }}$,

3. $\left[\mathfrak{X}_{1}, f \mathfrak{X}_{2}\right]_{E}=f\left[\mathfrak{X}_{1}, \mathfrak{X}_{2}\right]_{E}+\left(a\left(\mathfrak{X}_{1}\right) f\right) \mathfrak{X}_{2}-\left\langle\mathfrak{X}_{1}, \mathfrak{X}_{2}\right\rangle_{E} \mathscr{D} f, f \in C^{\infty}(M)$,

4. $\langle\mathscr{D} f, \mathscr{D} g\rangle_{E}=0, f, g \in C^{\infty}(M)$,

5. $a(\mathfrak{X})\left\langle\mathfrak{X}_{1}, \mathfrak{X}_{2}\right\rangle_{E}=\left\langle\left[\mathfrak{X}, \mathfrak{X}_{1}\right]_{E}+\mathscr{D}\left\langle\mathfrak{X}, \mathfrak{X}_{1}\right\rangle_{E}, \mathfrak{X}_{2}\right\rangle_{E}+\left\langle\mathfrak{X}_{1},\left[\mathfrak{X}, \mathfrak{X}_{2}\right]_{E}+\mathscr{D}\left\langle\mathfrak{X}, \mathfrak{X}_{2}\right\rangle_{E}\right\rangle_{E}$,

where $\mathscr{D}: C^{\infty}(M) \rightarrow \Gamma(E)$ is a map such that $\langle\mathscr{D} f, \mathfrak{X}\rangle_{E}=\frac{1}{2} a(\mathfrak{X}) f$.

\section{References}

[1] N. Hitchin, "Generalized Calabi-Yau manifolds," Quart. J. Math. 54 (2003) 281 [math/0209099 [math-dg]].

[2] M. Gualtieri, "Generalized complex geometry," DPhil thesis, math/0401221 [math.DG].

[3] C. M. Hull, “A Geometry for non-geometric string backgrounds,” JHEP 0510 (2005) 065 [hep-th/0406102].

[4] A. Dabholkar and C. Hull, "Generalised T-duality and non-geometric backgrounds," JHEP 0605 (2006) 009 [hep-th/0512005].

[5] C. M. Hull and R. A. Reid-Edwards, "Gauge symmetry, T-duality and doubled geometry," JHEP 0808 (2008) 043 [arXiv:0711.4818 [hep-th]].

[6] C. M. Hull and R. A. Reid-Edwards, "Non-geometric backgrounds, doubled geometry and generalised T-duality," JHEP 0909 (2009) 014 [arXiv:0902.4032 [hep-th]].

[7] N. Halmagyi, "Non-geometric String Backgrounds and Worldsheet Algebras," JHEP 0807 (2008) 137 [arXiv:0805.4571 [hep-th]].

[8] N. Halmagyi, "Non-geometric Backgrounds and the First Order String Sigma Model," arXiv:0906.2891 [hep-th].

[9] R. Blumenhagen, A. Deser, E. Plauschinn and F. Rennecke, "Bianchi Identities for Non-Geometric Fluxes - From Quasi-Poisson Structures to Courant Algebroids," Class. Quant. Grav. 29 (2012) 135004 [arXiv:1202.4934 [hep-th]].

[10] D. Mylonas, P. Schupp and R. J. Szabo, "Membrane Sigma-Models and Quantization of Non-Geometric Flux Backgrounds," JHEP 1209 (2012) 012 [arXiv:1207.0926 [hep-th]].

[11] R. Blumenhagen, A. Deser, E. Plauschinn, F. Rennecke and C. Schmid, "The Intriguing Structure of Non-geometric Frames in String Theory," Fortsch. Phys. 61 (2013) 893 [arXiv:1304.2784 [hep-th]].

[12] A. Chatzistavrakidis, L. Jonke and O. Lechtenfeld, "Dirac structures on nilmanifolds and coexistence of fluxes," Nucl. Phys. B $\mathbf{8 8 3}$ (2014) 59 [arXiv:1311.4878 [hep-th]].

[13] A. Chatzistavrakidis, L. Jonke and O. Lechtenfeld, "Sigma models for genuinely non-geometric backgrounds," JHEP 1511 (2015) 182 [arXiv:1505.05457 [hep-th]]. 
[14] D. Roytenberg, "Courant algebroids, derived brackets and even symplectic supermanifolds," Ph.D. thesis, math/9910078 [math.DG];

—-_, "A Note on quasi Lie bialgebroids and twisted Poisson manifolds," Lett. Math. Phys. 61 (2002) 123 [math/0112152 [math.QA]]

[15] D. Roytenberg, "AKSZ-BV Formalism and Courant Algebroid-induced Topological Field Theories," Lett. Math. Phys. 79 (2007) 143 [hep-th/0608150].

[16] M. Alexandrov, M. Kontsevich, A. Schwartz and O. Zaboronsky, "The Geometry of the master equation and topological quantum field theory,” Int. J. Mod. Phys. A 12 (1997) 1405 [hep-th/9502010].

[17] A. A. Tseytlin, "Duality Symmetric Formulation of String World Sheet Dynamics," Phys. Lett. B 242 (1990) 163;

—-_, "Duality symmetric closed string theory and interacting chiral scalars," Nucl. Phys. B 350 (1991) 395.

[18] W. Siegel, “Two vierbein formalism for string inspired axionic gravity,” Phys. Rev. D 47 (1993) 5453 [hep-th/9302036];

—-_, "Superspace duality in low-energy superstrings," Phys. Rev. D 48 (1993) 2826 [hep-th/9305073];

—-_, "Manifest duality in low-energy superstrings," In *Berkeley 1993, Proceedings, Strings '93* 353-363, and State U. New York Stony Brook - ITP-SB-93-050 (93,rec.Sep.) 11 p. (315661) [hep-th/9308133].

[19] C. Hull and B. Zwiebach, "Double Field Theory," JHEP 0909 (2009) 099 [arXiv:0904.4664 [hep-th]].

[20] O. Hohm, C. Hull and B. Zwiebach, "Background independent action for double field theory," JHEP 1007 (2010) 016 [arXiv:1003.5027 [hep-th]].

[21] O. Hohm, C. Hull and B. Zwiebach, "Generalized metric formulation of double field theory,” JHEP 1008 (2010) 008 [arXiv:1006.4823 [hep-th]].

[22] K. C. H. Mackenzie and P. Xu, “Lie bialgebroids and Poisson groupoids,” Duke Math. J. 73 (1994) 415.

[23] N. Ikeda, "Lectures on AKSZ Topological Field Theories for Physicists," arXiv:1204.3714 [hep-th].

[24] P. Bouwknegt, J. Evslin and V. Mathai, “T duality: Topology change from H flux," Commun. Math. Phys. 249 (2004) 383 [hep-th/0306062].

[25] P. Bouwknegt, J. Evslin and V. Mathai, "On the topology and H flux of T dual manifolds," Phys. Rev. Lett. 92 (2004) 181601 [hep-th/0312052].

[26] H. Bursztyn, G. R. Cavalcanti and M. Gualtieri, "Reduction of Courant algebroids and generalized complex structures,” Adv. Math. 211 (2007) 726 [math/0509640 [math.DG]].

[27] G. R. Cavalcanti and M. Gualtieri, "Generalized complex geometry and T-duality," A Celebration of the Mathematical Legacy of Raoul Bott (CRM Proceedings \& Lecture Notes) American Mathematical Society (2010) 341-366. [arXiv:1106.1747 [math.DG]].

[28] P. Ševera, "Poisson-Lie T-duality and Courant algebroids," Lett. Math. Phys. 105 (2015) 1689 [arXiv:1502.04517 [math.SG]].

[29] A. Chatzistavrakidis, A. Deser and L. Jonke, "T-duality without isometry via extended gauge symmetries of 2D sigma models,” JHEP 1601 (2016) 154 [arXiv:1509.01829 [hep-th]]. 
[30] A. Chatzistavrakidis, "Non-isometric T-duality from gauged sigma models," arXiv:1604.03739 [hep-th].

[31] I. Bakas, D. Lüst and E. Plauschinn, "Towards a world-sheet description of doubled geometry in string theory," arXiv:1602.07705 [hep-th].

[32] Z. J. Liu, A. Weinstein and P. Xu, "Manin Triples for Lie Bialgebroids,” J. Diff. Geom. 45 (1997) no.3, 547 [dg-ga/9508013]. 